Methodische Grundlagen für die Bewertung neuer Nutzungskonzepte für Produkte

\title{
Öko-Effizienz auf dem Prüfstand
}

\section{Strategien der Nutzungsdauerverlängerung und Nutzungsintensivierung von Produkten sind ein wichtiger Ansatz für die Reduzierung der Materialströme. Um die ökologischen Auswirkungen zu untersuchen, hat das IÖW erstmals einen konsistenten konzeptionellen Rahmen entwickelt.}

$\mathrm{W}$

Von Bernd Hirschl, Gerd Scholl und Frank Tibitanzl ährend derzeit eine Entkopplung des Wirtschaftswachstums vom Umweltverbrauch beobachtbar ist (1), nimmt gleichzeitig der absolute Pro-Kopf-Verbrauch an stofflichen Ressourcen in Ländern wie Deutschland, Japan, USA und den Niederlanden nach wie vor zu. Von einer "Dematerialisierung" industrieller Wirtschaftsweisen kann bisher nicht die Rede sein. Diese Entwicklung wird auch am Beispiel verschiedener Konsumgüter wie Waschmaschinen, PCs oder Fernseher sichtbar, bei denen die produktspezifischen Effizienzgewinne durch ein überproportionales Mengenwachstum aufgefressen werden. Beispielsweise ist der spezifische Energiebedarf eines Farbfernsehgerätes 1995 auf 30\% des Wertes von 1970 gesunken. Gleichzeitig hat sich der Gesamtenergieverbrauch aller Fernsehgeräte im selben Zeitraum verdoppelt (2).

Es muß Ziel einer Politik der Nachhaltigkeit sein, zum Schutz der Ökosphäre die Mengenströme, die mit der vorherrschenden Wirtschaftsweise in den Industrieländern einhergehen, deutlich zu reduzieren. Dies können Strategien der Nutzungsintensivierung (Verringerung der Stoffströme) und Nutzungsdauerverlängerung (Verlangsamung der Stoffströme) leisten (3).

\section{Konsistente Terminologie no̊tig}

Die fast exponentiell wachsenden Forschungsaktivitäten und Veröffentlichungen zu diesem Thema haben $\mathrm{zu}$ einer steigenden Begriffsvielfalt und damit zwangsläufig auch Begriffsverwirrung gefiihrt. Zentrale Säulen dieser Begriffswelt sind Ausdrücke wie „neue Nutzungskonzepte“, ,ökoeffiziente Dienstleistungen “, ,ökointelligente Produkte" und „Funktionsorientierung", die teilweise synonym, teilweise in sehr unterschiedlichen Kontexten verwendet werden. Ein geeigneter Ausgangspunkt für die Entwicklung eines konsistenten und operationalisierbaren Begriffsap- parats ist die Definition der beiden Strategiedimensionen "Nutzungsintensivierung“ und „Nutzungsdauerverlängerung" (4).

Innerhalb einer Verfügungsdauer, aber auch über den gesamten Zeitraum der Nutzungsdauer steht der Auslastungsgrad des Produktes im Blickpunkt unseres Interesses. Dieser läßt sich als Nutzungsintensität (NI) eines Produktes quantifizieren. Damit auch parallele, gemeinschaftliche Nutzungen erfaßt werden können, wird die Nutzungsintensität nicht als Verhältnis von Zeitdauern, sondern in Leistungseinheiten pro Produkteinheit angegeben (5). Sie kann daher ganz allgemein wie folgt definiert werden:

$$
\mathrm{NI}=\mathrm{L} / \mathrm{P}
$$

Mit L bezeichnen wir die Leistungseinheiten (z.B. Personenkilometer), die von einem/einer Produkt(menge) P (z.B. Pkw), während der Nutzungsdauer abgegeben werden. Unter Nutzungsintensivierung verstehen wir folglich eine Erhöhung der Nutzungsintensität, d.h. eine betragsmäßige Erhöhung von NI. Eine Nutzungsintensivierung kann einerseits durch eine Erhöhung der Leistungsabgabe bei unveränderter Produktmenge und andererseits durch die Reduktion der Produktmenge bei gleichbleibender Leistungsabgabe erreicht werden.

Der Begriff Nutzungsdauerverlängerung (NV) beschreibt die zeitliche Ausdehnung der Nutzungsdauer eines Produktes. Diese kann - bei konstanter Lebensdauer - auf zwei Wegen erreicht werden. Zum einen kann die Nutzungsdauer durch eine Erhöhung der Anzahl an Verfügungsdauern bzw. Nutzern, zum anderen durch die Verlängerung eines Verfügungszeitraumes erfolgen. Darüber hinaus wird durch eine technische Lebensdauierverlängerung eine Ausweitung der Nutzungsdauer eines Produktes ermöglicht. Tabelle 1 gibt einen Überblick über die definierten Begriffe und nennt jeweils beispielhafte Ansätze.

Fast alle Veröffentlichungen zum Thema ,neue Nutzungskonzepte für Produkte“ oder ,„̈kologi- sche Dienstleistungen" transportieren in der einen oder anderen Form den folgenden Gedankengang: Es sind nicht Sachgüter, die im Vordergrund des Interesses eines Konsumenten stehen, sondern der Nutzen, den diese stiften. Da es aber unterschiedliche Möglichkeiten gibt, die menschlichen Bedürfnisse $\mathrm{zu}$ befriedigen und damit Nutzen zu stiften, existiert also mindestens eine Alternative, die mit dem geringsten Stoffund Energieeinsatz und den niedrigsten humanund ökotoxikologischen Risiken verbunden ist.

\section{Ökologische Wirkungen never Nutzungskonzepte}

Daraus läßt sich die Ausgangsthese ableiten, daß NV/NI-Strategien zu einer Steigerung des Leistungspotentials eines Produktes bzw. zu seiner höheren Auslastung beitragen. D.h. eine gegebene Funktion kann mit einem reduzierten Materialund Energieaufwand erfiillt werden, wodurch die Ressourcenproduktivität wächst. Aus der Perspektive einer ganzheitlichen ökologischen Bewertung sind NV/NI-Strategien also dann ökologisch vorteilhaft, wenn sie die Ressourcenproduktivität erhöhen, ohne daß sich andere ökologische Parameter, z.B. Flächenverbrauch, Biodiversität oder Schadstofffracht, verschlechtern.

Hinsichtlich der ökologischen Wirkungen von NV/NI-Strategien unterscheiden wir leistungsspezifische Wirkungen von Niveaueffekten. Unter dem leistungsspezifischen ökologischen Effekt (bzw. der ökologischen Effizienz) von NV/NI-Strategien verstehen wir dabei die von einer intensiveren und/oder längeren Nutzung ausgehende Veränderung der Umweltbelastung pro Leistungseinheit. Als Niveaueffekt wird die Veränderung der Umweltbelastung bezeichnet, die allein auf einer Veränderung der Leistungsnachfrage basiert.

Zur besseren Veranschaulichung dieser beiden Effekte ist es hilfreich, die gesamte Umweltbelastung (U) einer spezifischen Nutzungsform in zwei Komponenten zu zerlegen:

$$
\mathrm{U}=\mathrm{U} / \mathrm{L} \cdot \mathrm{L}=\mathrm{u} \cdot \mathrm{L}
$$

Die gesamte Umweltbelastung (U) entspricht demnach der Umweltbelastung pro Leistungseinheit (ökologische Effizienz $u=U / L$ ) multipliziert mit der Zahl der nachgefragten Leistungseinheiten (Leistungsniveau L).

\section{Anwendungsbeispiel Auto}

Wenn man beispielsweise annimmt, daß die einzige von Autos ausgehende Umweltbelastung im Rohstoffverbrauch (Erdöl) besteht, welche dann bei einer durchschnittlichen Lebensdauer von 
zehn Jahren und einer durchschnittlichen jährlichen Fahrleistung von 50.000 Personenkilometern im Jahr 200.000 Liter beträgt, dann ergibt sich ein u von 0,4 Liter/Personenkilometer.

Hinsichtlich der ökologischen Effizienz, d.h. des leistungsspezifischen Effekts, sind drei verschiedene Fälle möglich (Abbildung 1):

a) Die Umweltbeanspruchung pro Leistungseinheit sinkt, beispielsweise bei der Mitnahme zusätzlicher Personen, wenn der Verbrauch an Betriebsstoffen eindeutig unterproportional zur Personenzahl steigt (6).

b) Die Umweltbeanspruchung pro Leistungseinheit bleibt konstant, so zum Beispiel bei einer durch die Bauweise bedingten Verdoppelung der Lebensdauer, die das Gewicht ebenso annähernd verdoppelt.

c) Die Umweltbeanspruchung pro Leistungseinheit steigt, so zum Beispiel bei einer Lebensdauerverlängerung, die nicht nur das Gewicht erhöht, sondern auch zur Verwendung zusätzlicher technischer „Features“ führt (Klimaanlage, elektrischer Fensterheber, etc.), so daß der ökologisch günstige Effekt der höheren Lebensdauer überkompensiert wird.

Natürlich zielt die Implementierung von NV/NIStrategien zunächst immer auf eine effizientere Nutzung der Umweltressourcen, d.h. eine höhere ökologische Effizienz, ab. Entscheidend für die ökologische Bewertung ist aber der Gesamteffekt, d.h. es muß auch beachtet werden, welche Auswirkungen der Übergang zu NV/NI-Strategien auf die Leistungsnachfrage (Niveaueffekt) hat. Hierbei sind drei Fälle relevant:

1) Eine Verbesserung der ökologischen Effizienz kann durch einen positiven Niveaueffekt verstärkt werden. So fahren zum Beispiel Mitglieder von Car-Sharing Organisationen weniger Kilometer pro Jahr als vor ihrem Beitritt, weil die höheren Transaktionskosten der Nutzung zu einer bewußteren Reflexion der eigenen Mobilitätsbedürfnisse führen (7).

\section{Abb. 1: Umweltwirkungen von NV/NI}

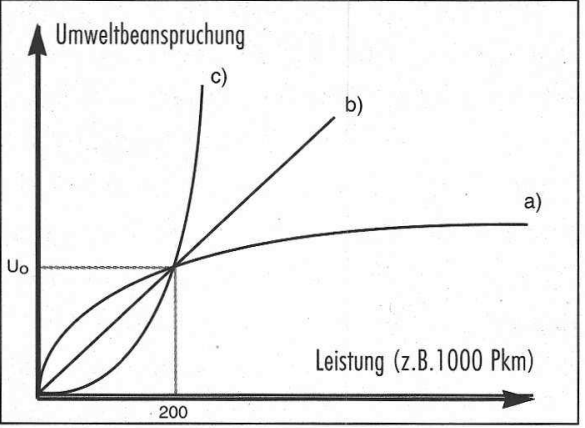

Quelle: eigene Darstellung

\begin{tabular}{l|l|l|l|l|l|}
\hline \multicolumn{2}{|c|}{ Tabelle I: Nutzungsintensivierung und Nutzungsdauerverlängerung } \\
\hline Nutzungsintensivierung (NI)
\end{tabular}

2) Auf der anderen Seite können durch die Umsetzung von NV/NI-Strategien neue Konsummöglichkeiten entstehen, so daß die Nachfrage nach Leistungseinheiten insgesamt zunimmt. So wäre Car-Sharing beispielsweise ökologisch kontraproduktiv, wenn hierdurch die Mobilitätsnachfrage deutlich steigt und positive Effekte überkompensiert werden, z.B. durch solche Nutzer, die vorher überhaupt kein Auto besessen haben. Dies ist dann der Fall, wenn L prozentual schneller steigt als u sinkt.

3) Schließlich kann die Leistungsnachfrage unverändert bleiben, so daß die Gesamtwirkung allein auf eine verbesserte ökologische Effizienz zurückzuführen ist.

Die methodischen Ausführungen machen zum einen deutlich, daß ,moving from products to services and extending product durability will not always reduce resource use and environmental impacts“ (8), weisen zum anderen aber auch darauf hin, daß gerade mit Blick auf die Niveaueffekte NV/NI-Strategien ein großes ökologisches Entlastungspotential in sich bergen.

\section{Anmerkungen}

(1) World Resources Institute et al. (Hrsg.): Resource Flows. The Material Basis of Industrial Economies, Washington D.C. 1997, S.2.

(2) Behrendt, Siegfried: Paradigmenwechsel in der Produktpalette, in: Zukünfte, Nr. 20 (1997), S. 16-17.

(3) Die Begriffe und maßgebliche konzeptionelle Fortschritte gehen vor allem auf die Arbeiten von Stahel zurück, vgl. z.B. Stahel, Walter: Allgemeine Kreislauf- und Rückstandswirtschaft. Intelligente Produktionsweisen und Nutzungskonzepte, Karlsruhe 1996.

(4) Die Entwicklung einer konsistenten Nomenklatur und ferner die Erfassung und Analyse der EinfluBfaktoren auf die Nutzungsmuster von Produkten sind die Ziele der ersten
Arbeitsphase des IÖW-Projekts „Neue Nutzungskonzepte für Produkte - Ökologische Entlastungspotentiale, Umsetzungsprobleme und Entwicklungsperspektiven von Strategien zur Nutzungsdauerverlängerung (NV) und Nutzungsintensivierung (NI)" ${ }^{\prime}$.Der Zwischenbericht des vom Bundesministerium für Bildung, Wissenschaft, Forschung und Technologie geförderten Vorhabens, wird als IÖWSchriftenreihe erscheinen. Weitere Informationen unter http://www.ioew.de/projekte.

(5) Die Nutzungsintensität läßt sich z.B. als Verhältnis der Gebrauchszeit zur Verfügungszeit angeben, womit jedoch parallele Nutzungen $-z . B$. gemeinschaftliche Autonutzung - nicht erfaßt werden.

(6) Hierzu müßte man theoretisch noch das Umweltprofil der Vermittlungsdienstleistung einer Miffahrzentrale addieren. Denn auch dieses ist mit einem Ressourceninput verbunden (Kommunikationstechnologie, Papier, etc.), der jedoch aufgrund der hohen Vermittlungsziffern kaum zu Buche schlagen dürfte.

(7) Vgl. Meijkamp, Rens: Changing Consumer Needs by Eco-efficient Services. In: The Centre for Sustainable Design (Hrsg.): Towards sustainable product design. London 1997, und Baum, Herbert, Stephan Pesch: Untersuchung der Eignung von Car-Sharing im Hinblick auf die Reduzierung von Stadtverkehrsproblemen. Forschungsbericht FE-Nr. 70421/93, Köln 1994.

(8) OECD (Hrsg.): Eco-Efficiency, Paris 1998.

\section{Die Autoren}

Bernd Hirschl, Gerd Scholl und Frank Tibitanzl sind wissenschaftliche Mitarbeiter im Forschungsfeld Öko logische Produktpolitik des IÖW.

Kontakt: IÖW Heidelberg, Bergstraße 7 , 69120 Heidelberg, Tel. 06221/ 64916-0, Fax 06221/ 27060,

E-mail: mailbox@ioew.hd.eunet.de 
(c) 20I0 Authors; licensee IÖW and oekom verlag. This is an article distributed under the terms of the Creative Commons Attribution Non-Commercial No Derivates License (http://creativecommons.org/licenses/by-nc-nd/3.o/), which permits unrestricted use, distribution, and reproduction in any medium, provided the original work is properly cited. 